\title{
Citizen Engineering: Evolving OSS Practices to Engineering Design and Analysis
}

\author{
Zhi Zhai, Tracy Kijewski-Correa, Ashan Kareem, \\ David Hachen, and Gregory Madey \\ University of Notre Dame, Notre Dame, IN-46556, USA \\ \{zzhai, tkijewsk, akareem, dhachen, gmadey\}@nd.edu
}

\begin{abstract}
Open Source Software (OSS) development has much in common with concepts such as crowdsourcing, citizen science, collective intelligence, human-based computation, and what we call "Citizen Engineering (CE)". We report on several pilot projects that apply these shared principles of OSS development to engineering activities beyond software engineering. CE models harness human computing power from open communities, which commonly consist of a cohort of geographically and/or institutionally scattered citizens - professionals or amateurs - to collaboratively solve real-world problems. In most cases, the problems targeted are challenging to computers, but manageable or trivial to human intelligence. In these systems, while humans play fundamental roles, whether they are project architects or problem solvers, the implementation of $\mathrm{CE}$ is greatly facilitated by the advance of information technology, particularly the Internet, considered as "creative mode of user interactivity, not merely a medium between messages and people" 10. In this paper, we characterize existing citizen engineering practices into 6 major categories, followed by a discussion of 4 ongoing projects, aiming to provide new perspectives and insights for achieving successful $\mathrm{CE}$ project designs.
\end{abstract}

\section{Introduction}

Open Source Software (OSS) development is often researched for its novel approaches to software engineering. OSS is typically characterized by its openness, distributed and often voluntary participation, and end-user participation in the software engineering processes. It is observed that similar open, distributed, possibly voluntary and end-user based engineering activities are emerging under various labels, such as crowdsourcing, end-user participation in the design process, citizen science, collective intelligence, human-based computing, and what we call Citizen Engineering. Evolving information technologies provide unprecedented opportunities to solicit contributions from the public, i.e., citizens. Two popular examples are Wikipedia and YouTube, where regular citizens can freely contribute and evaluate contents as long as they abide by certain community rules. Advancing cyber-infrastructure, such as high speed networks, increasing computational capabilities, and high capacity databases, enables transformative

I. Hammouda et al. (Eds.): OSS 2012, IFIP AICT 378, pp. 61-77, 2012.

(C) IFIP International Federation for Information Processing 2012 
platforms to diminish the barriers among geographically or institutionally dispersed users. People can easily channel their brainpower and "cognitive surplus" to accomplish meaningful work for a common good, largely in their spare time 25]. Characterizing the existing CE projects, we identify 6 major categories:

1. Crowd Decisions: Exemplified by the Reddit and Digg reader voting systems, by casting their votes, crowds have the capacity to collectively identify high quality products.

2. Crowd Sumbission/Funding/Journalism: Individuals in the crowd can make directed contributions, which could take the form of submitting a piece of content, chipping in a small amount of money, or reporting on what one has heard, witnessed, or interpreted. Together, pieces of contributions are channeled and possibly merged, and results are either fed back to serve community interests or stir up broader social attentions.

3. Crowd Wisdom: Networks of organized participants contribute their knowledge in specific areas, oftentimes leading to elaborate artifacts, e.g., the Mozilla web browser, considered as suitable alternatives for proprietary counterparts.

4. Crowd Byproduct: Standalone and Piggyback are the two major types 13 . In standalone systems, users contribute human-based computation as byproducts of major activities, e.g. Biogames 22. Piggyback systems collect "user traces" generated out of other purposes to solve target problems [20]. For instance, in search engine optimization, Google records users' query history to prompt search keywords and suggests spell corrections later on.

5. Micro Task: Certain tasks can be divided into small units and assigned to online workers. Such small units of work usually require lower human skills, and their results are easy to merge. The online platforms, such as Amazon Mechanical Turk and Crowdflower, provide such services.

6. Innovation Tournament: Outside human resources can be harnessed via open challenges or competitions. If the ideas/inventions get adopted by the institutions seeking solutions, winners can be recognized by monetary rewards, non-monetary acclaims or both, e.g., the DARPA red-balloon competition [26].

Following the 6 categories above, we present 4 pilot $\mathrm{CE}$ projects in this paper, which come from a larger NSF funded study - Open Sourcing the Design of Civil Infrastructure [19]. These 4 pilot projects are: Haiti Earthquake Photo Tagging (Micro Task); Smart Phone Infrastructure Monitoring (Crowd Submissions); OpenFOAM Simulation (Crowd Wisdom), focused on citizen engineering requiring a high level of expertise; Shelters For All (Innovation Tournament). In Sections II-V, we discuss them in detail.

In addition to the 6 categories, we classify CE projects along 3 dimensions for a deeper understanding of CE implementation: 1) Contributor Motivation, 2) Human Skills Required - how tasks get fulfilled, and 3) Quality Evaluation - how results get evaluated. Fig. 1) shows how the 4 projects are positioned in this 3-dimensional feature space. 


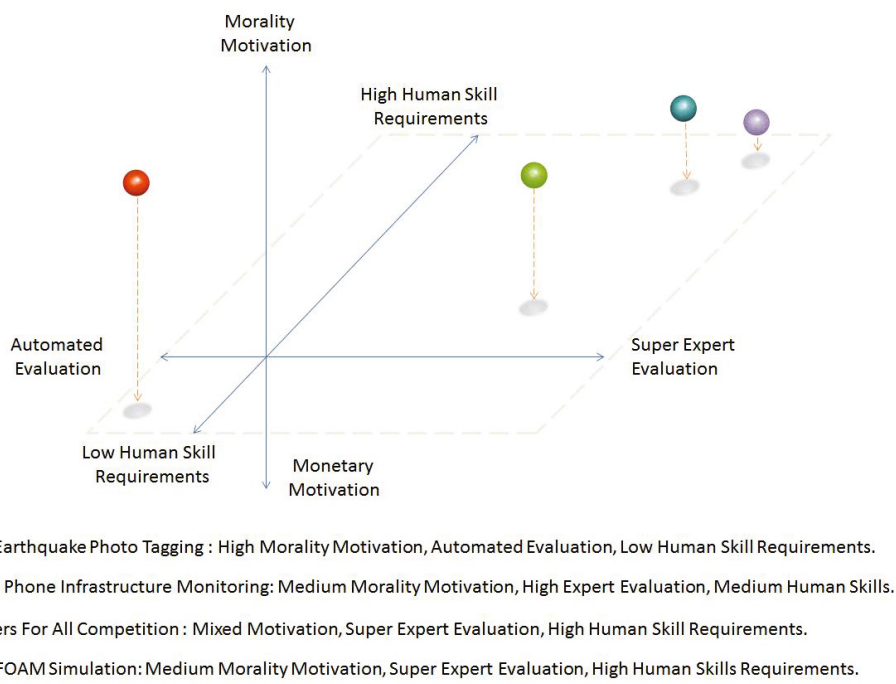

Fig. 1. 3-Dimensional Classification of CE Projects: Motivation, Skill Level and Evaluation

\section{Project I: Haiti Earthquake Photo Tagging}

After the 2010 catastrophic Haitian earthquake, to help local residents rebuild their homeland, civil engineers from University of Notre Dame visited Haiti and took thousands of photos of impaired buildings, intending to classify common damage patterns, and thus to inform redesign and rebuilding efforts [23]. However, the volume of earthquake photos surpassed their capacity to process. To answer this call, a collaborative team of computer scientists, civil engineers, and sociologists built a CE platform, where crowds could be harnessed to fulfill photo classification tasks.

For this pilot project, students were recruited using announcements on mailing lists and school-wide posters, resulting in 242 students participating in the experiment as surrogates for citizen engineers. Their online activities were recorded in detail, including photo tagging classifications, the time spent tagging each photo, and login/logout timestamps.

Over 17 days, the crowd submitted 9318 photo classifications on 400 sample photos. The photo taggers came from a broad range of backgrounds - some of the participants were engineering and science majors, others were from finance, history or other humanities - and hence classifications were at times widely divergent. This heterogeneity mimics what is commonly observed in CE projects: highly diverse education levels of the users and variable quality of task fulfillment.

Positioning this project into the 3-dimension space: 1) contributors in the system were highly motivated by moral responsibilities, 2) the online crowds input their opinions about the images and collectively produced crowd consensus 


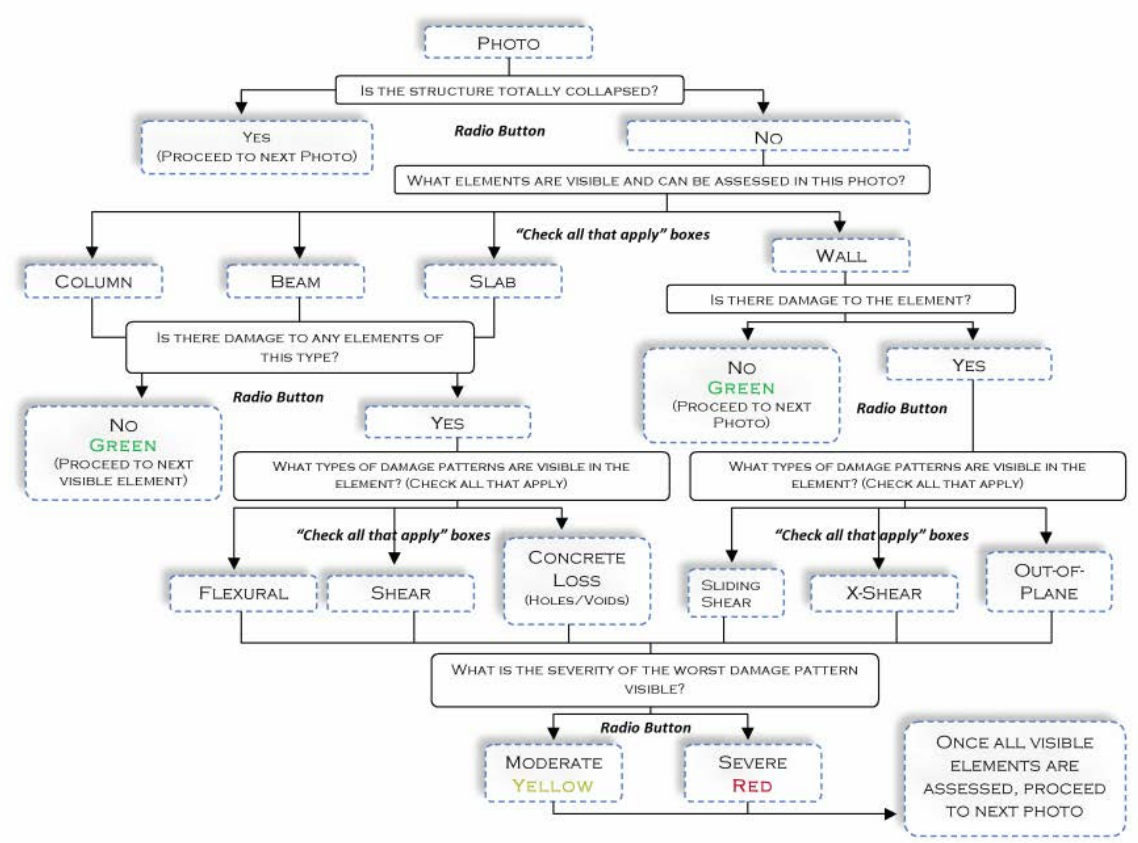

Fig. 2. Classification schema. As crowd went deeper along the tree, their answers diversified.

for organizers to consider, without expert evaluation involved, and 3) high-level civil engineering knowledge was not a prerequisite, since users could acquire required skills through tutorials provided by the platform.

\subsection{Workflow Description [27]}

Upon agreeing to terms and conditions on the front page, subjects were directed to a sign-up page, and asked to create their login credentials.

1. Registration. After subjects logged into the website, they saw a consent form with a brief description of the experiment: the task was to classify the types and serverity of earthquake damage depicted in 400 photos.

2. Entry Survey. The purpose of this questionnaire was to collect demographic and attitudinal data from the subjects.

3. Tutorials. The tutorial provides detailed information about how to properly classify the damage depicted in a photo, and by using hyper-links, subjects can return to this tutorial during their tagging process.

4. Damage Classification. To classify photos, subjects received a single, randomly chosen photo at a time, until they completed all 400 photos in the system or their allocated time period expired. 
5. Exit Survey. At the end of a 7-day period, subjects were asked to complete a brief exit survey. The survey has questions like the motivation driving subjects to allocate time for voluntary work, the difficulty in classifying photos, the degree to which they found the task was interesting, etc.

\subsection{Tagging Questions}

As shown in Fig. 2] to classify a photo, subjects followed a 5-step damage assessment process. These steps are:

1. Image Content. Determine if an entire structure or only a part of the structure is visible in the image.

2. Element Visibility. Identify which elements (beams, columns, slabs, walls) of the building are visible and can be assessed.

3. Damage Existence. For each of these visible elements, determine if any of those elements are damaged.

4. Damage Pattern. For each of the elements identified as damaged, identify the damage pattern.

5. Damage Severity. For each of the elements identified as damaged, assess the severity of the damage (Yellow or Red).

Since there are at most 25 classification questions asked for each photo, maximumly, a user can earn 25 points from it. If the crowd consensus is that there is no damage on a certain element of the building, we do not further consider users' inputs about the damage pattern and severity of that building element. Compared to the similar image classification work conducted in [3], this workflow presents a comprehensive photo tagging schema with potential to generate new knowledge because of its depth.

Similar to Open Source Software development, when the end users are not developers themselves, the factors of human computer interaction (HCI) should be taken into consideration at early stage of development [17. In this regard, we especially emphasized the web interface's friendliness and tutorials' clearness when developing the system.

\section{Project II: Photo Sensing on Deteriorating Infrastructure}

In United States, deteriorating infrastructure is in a worrying condition (See Fig. 3), and may lead to tragic disasters. In 2007, the busy I-35W Bridge in Minneapolis, MN, USA, collapsed during evening rush hour, claiming lives of 13 and injuring another 145, besides financial losses. In retrospect, this bridge had evidence of crumbling and corrosion before it collapsed. This may suggest that this type of accidents can be prevented if signs and traces indicating infrastructure deteriorating is timely reported to corresponding authorities.

Human sensing systems leverage a large number of volunteers to conduct mobile sensing, which can be a complement to traditional inspection procedures. 


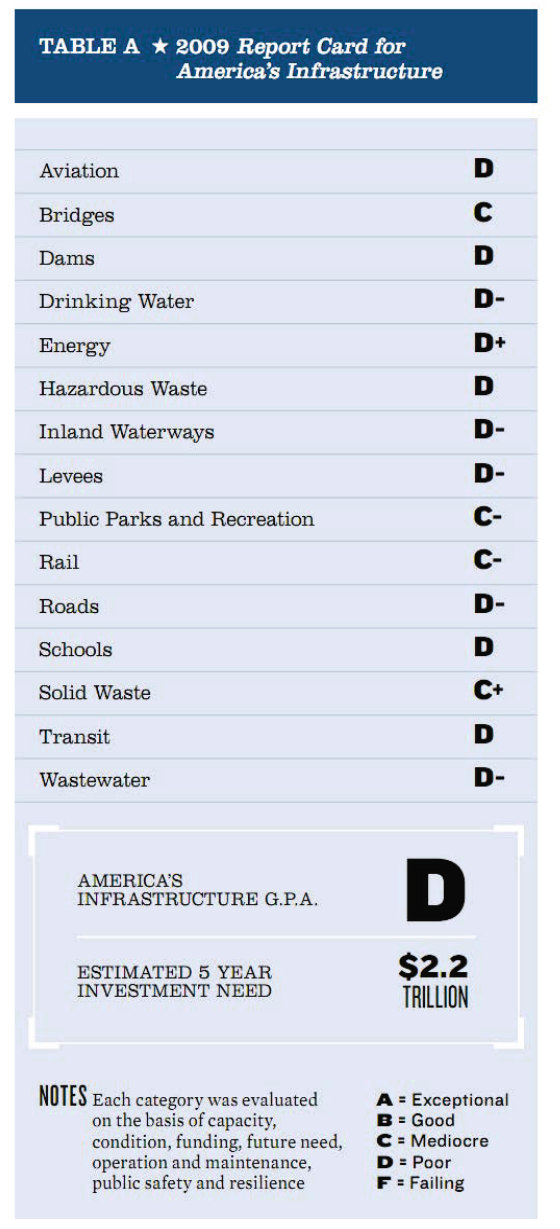

Fig. 3. Grades of American Infrastructure. Sources: American Society of Civil Engineers [1].

Given the increasing pervasiveness of 5.81 billion mobile phone subscribers [5], portable digital devices across the globe have evolved into a pervasive sensor platform, creating the possibility of organizing phone holders as human-based sensors [18. This "human sensor network" [16] [12], composed of digital devices and human subscribers, has demonstrated advantages compared to traditional sensor systems:

1. Hand-held digital devices, due to high penetration, have better coverage and flexibility. The resulting data, which are crumbling infrastructure photos in our case, provide significantly more informative insights.

2. New apps/add-ons/plug-ins can be timely installed and periodically updated by human users carrying the devices. 
3. In a human-based system, each mobile device is associated with a phone holder, whose assistance could be leveraged to achieve complex functionality. For example, residents usually have intimate knowledge of patterns and anomalies in their communities and neighborhoods [11].

\subsection{Components and Considerations}

Inspired by previous practices [2] 24] 15], we piloted an infrastructure monitoring system. The workflow is shown in Fig. 4, and the following 9 components comprised the workflow:

1. User Recruitment. Citizen inspectors in this experiment were college juniors and sophomores. As their hometowns are widely spread across the country, collectively they have good coverage of the nationwide civil infrastructure.

2. User Education. Research in psychology shows that individuals motivated by goals that are both well-specified and challenging tend to have higher levels of effort and task performance than goals that are too easy or vague [21. In design, we strive to provide a well-instructed and easy-to-follow tutorial.

3. Information Recording. Participants were encouraged to go outdoors and snap photos of problematic infrastructure, such as cracked structures, crumbling concrete, broken piers, and leaking tunnels. As photo-taking functionality is built in most digital devices, there is no need to develop new applications in this study.

4. Data Sending. Two options were provided for photo submission, as shown in Fig. 5 ,

- If the user has any type of smart phones equipped with the geo-tagging function, s/he can email us photos directly or upload photos via web interface. Our software can automatically retrieve geo-coordinates from the submissions.

- If the digital device cannot geo-tag the photo automatically, users can either input street address or use a movable marker to pinpoint the location on a Google Map embedded in the uploading page (See Fig. 6).

5. Data Collecting. A data repository hosts the web service, receiving data form the digital devices through different approaches. A MySQL database saves the metadata of each photo into database tables.

6. Data Processing. Data access was managed according to terms and conditions agreed by participants. The intricacy and importance of data security and privacy should be always emphasized [11. This is a two-fold issue: (1) Privacy concerns: over time, timestamps on photo submissions, combined with geo-space information, provide traceable data about citizen inspectors' life patterns; (2)Homeland security: the weak points of the national infrastructure may become targets of potential terrorist attacks. In this regard, 


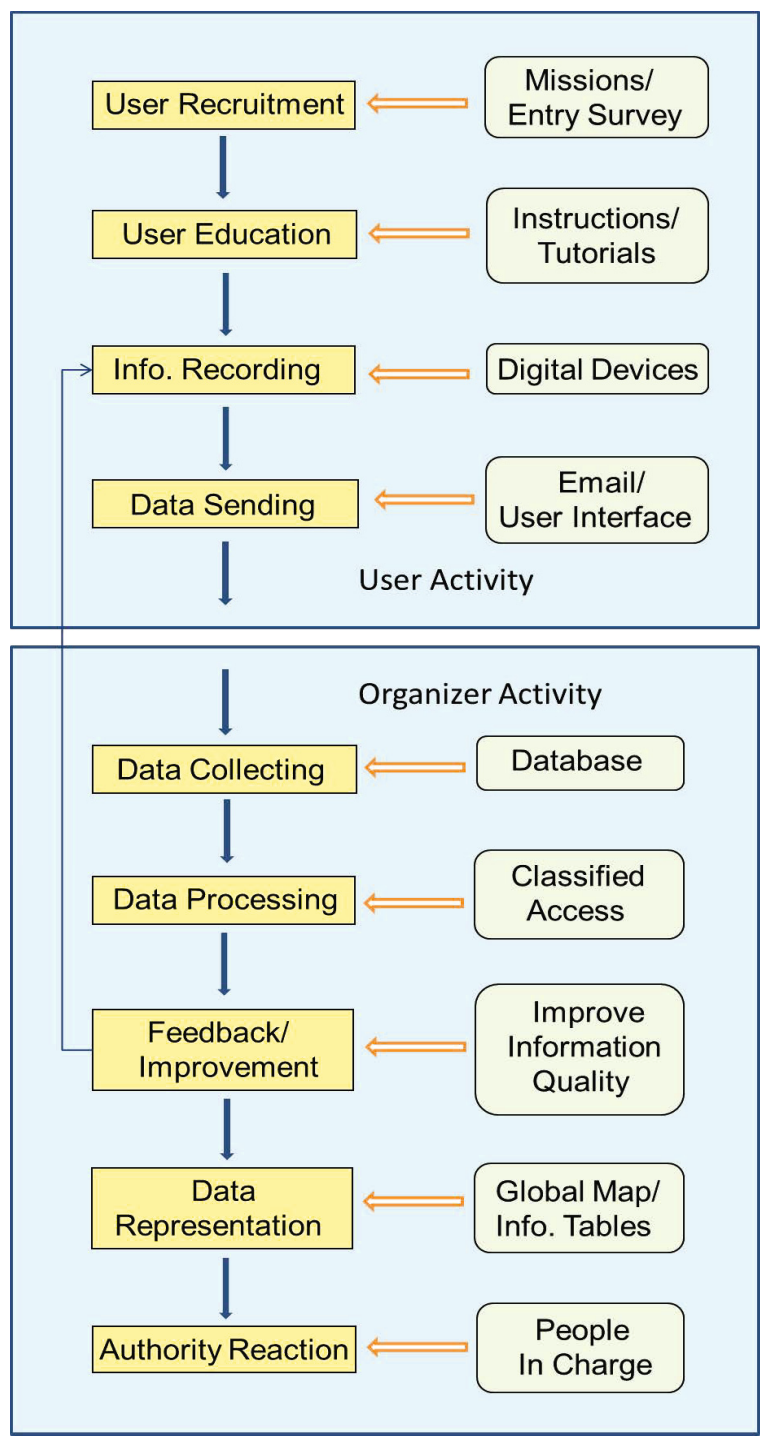

Fig. 4. Framework of Participatory Sensing

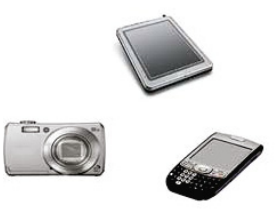

Digital Devices

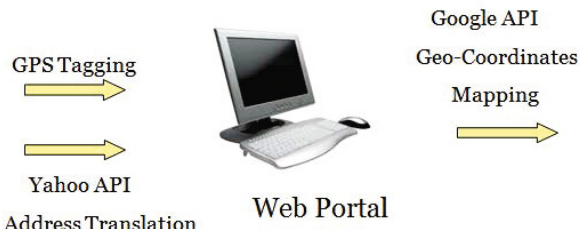

Web Portal

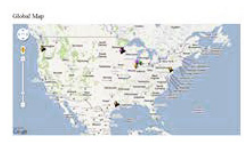

Google Global Map

Fig. 5. Two Options for Photo Submission 
the protection policy on our experimental portal was that any photos coming from an individual were only visible to that individual. The global map, where overall infrastructure photos were aggregated and presented, was only visible to project organizers, masked off from the public.

\section{Feedback and Improvement}

If data was found missing, the user could revisit the venue and retrieve complement data, possibly from variable angles and different distances, thanks to the human intelligence associated with the digital device.

\section{Data Presentation}

Aggregated data was visualized with color balloons on a global map, with each color balloon representing one damaged infrastructure photo, as shown in Fig. 7 .

\section{Policy Influence}

Relevant authorities can be reached and informed in the wake of possible disruptive development of inspected infrastructure, and the location and severity of deterioration can be further investigated to a finer resolution if necessary.

\subsection{Result and Discussion}

In a period of 12 days, we received 170 photos from 25 users, covering 30 cities/townships across 6 states in US. Most photos identified deteriorating infrastructure, with a large portion of submissions in fairly high quality (6 sample photos are shown in Fig. 8). This study provides a new perspective, where the $\mathrm{CE}$ approach can be leveraged to enhance human ability in the area of detecting infrastructure flaws, reducing financial resources, and more importantly saving lives.

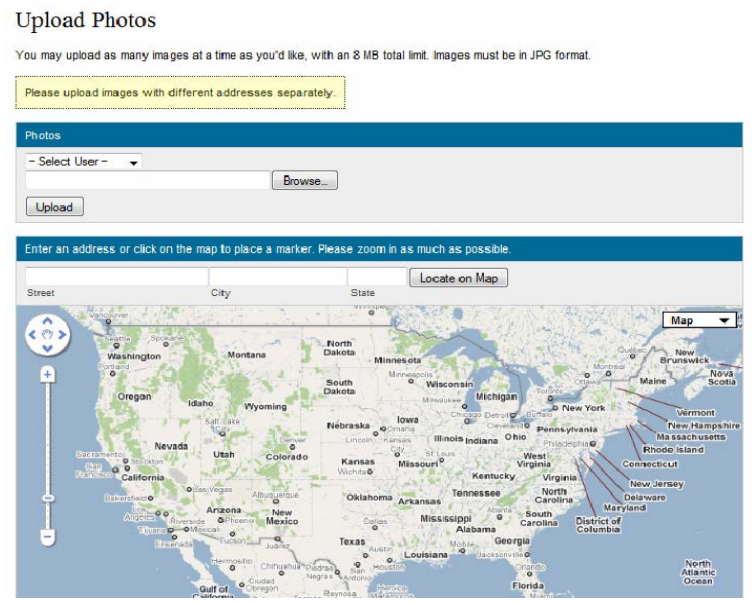

Fig. 6. Two Uploading Options: Street Fields Vs. Map Markers 


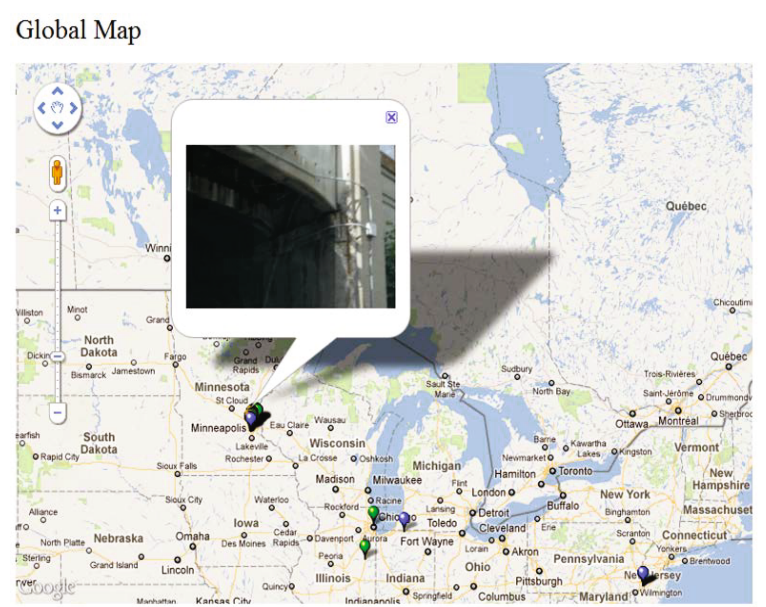

Fig. 7. Global Map: Data Representation and Visualization

\section{Project III: Shelters for All Competition - Safe and Affordable Housing for the Developing World}

When organizations encounter limited human resources to solve a challenging problem, they can pursue ideas outside the organization via open calls, namely innovation tournaments. Examples falling into this category include NetFlix Prize 8] for movie recommendations and IBM Innovation Jam 9] for sale improvement.

Enlightened by these successful experiments, we initiated a new innovation challenge, titled "Shelters For All Competition". By conducting this open competition, we aimed to achieve two goals (1) soliciting feasible designs of affordable housing in underdeveloped regions throughout the world; (2) assessing the pros and cons of the innovation tournament model in organizing crowdsourcing work.

\subsection{Background}

Fifteen of the twenty most populated cities in the world are currently located in developing countries, reflective of a wider trend that the majority of the world's population are increasingly hosted in urban zones. This results in densely populated, unstructured settlements or slums, the lack of safe drinking water, proper sanitation and other basic necessities in such landscapes, and other social issues.

Recognizing the need for housing innovations, this competition was designed to tap into the creativity of the public - individuals and teams - to design lowcost, safe housing to the world's urban poor. While adoption and sustainability by a target country or region is important, it is hoped that innovative solutions solicited from global community can have broader applications. To effectively 
Minneapolis
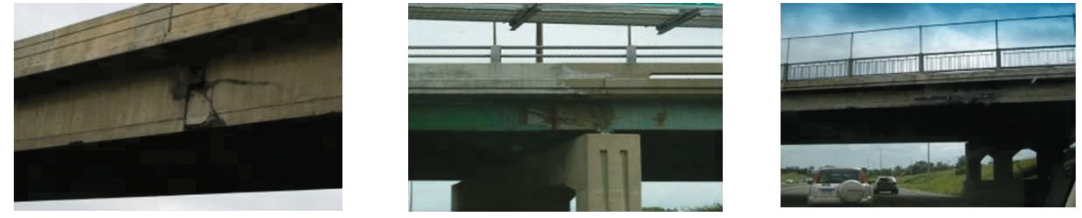

Portland
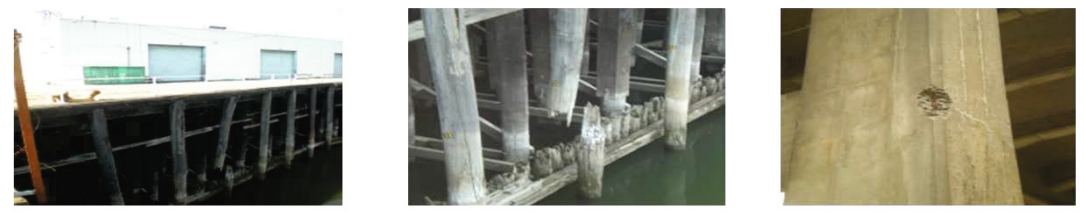

Fig. 8. Sample Photos from User Submissions

meet the goals of improving living conditions of developing countries, desirable designs should have the following properties:

1. Resiliency to insure life-safety and protection against natural disasters and other environmental factors.

2. Feasibility to be practically implemented using locally available technologies, capabilities and materials.

3. Sustainability to be supported indefinitely using local resources (economic \& natural), technologies \& skills of the community, and can adapt to their evolving needs.

4. Viability to earn the support of most local stakeholders as culturally appropriate, so that ideas are not just accepted, but embraced and promoted.

5. Scalability to be applied elsewhere beyond the particular country or region used for solution development.

The welcome page of the competition platform is shown in Fig. 9, and competition prizes and awards are designed as:

1. The grand prize $\$ 10,000$, granted to the best design among all submissions.

2. Popular vote award $\$ 1,000$, awarded to the submission that obtains the highest score in peer reviews.

3. Referral award $\$ 600$, distributed to the 3 individuals whose referrals result in the most submissions. 


\section{Shelters for All}

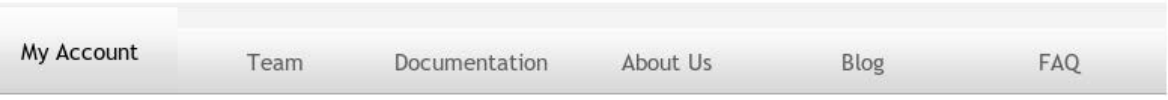

\section{My Account}

\section{Account Progress}

Fig. 9. User Interface of Shelters For All Competition Website

\subsection{Results}

By the time we closed the submissions site on Jan. 22, 2012, there were 99 valid solutions from 26 teams and 73 individuals. Most designs reflected participants' unique perspectives and considerations on tackling the challenge.

Similar to OSS projects, participants in this open competition are global citizens, with dramatically different working schedules and physical facilities. For them, the major connecting point and the information source are the project website. OSS experiences tell us that even a very short period of downtime on the server side would frustrate a certain number of participants. As such, as competition proceeded, we made particular emphasis on the system stability and scalability.

\section{Project IV: Expert-Citizen Experiment - OpenFOAM Simulation}

To design a successful Citizen Engineering system, an inevitable challenge is that the contributors, i.e., Citizen Engineers - professionals, researchers, students, and even the public at large - usually have a broad range of expertise and talents, since individuals are at various stages in their careers. Among them, there is a certain portion of well-trained professionals, who have received formal training and/or have years of practical experience. While many engineers are intrinsically motivated to provide voluntary service to society, for licensed engineers, Professional Development Hours (PDHs) are necessary to maintain licensure, and as such there may be pragmatic incentives for licensed engineers to engage in citizen engineering activities. 


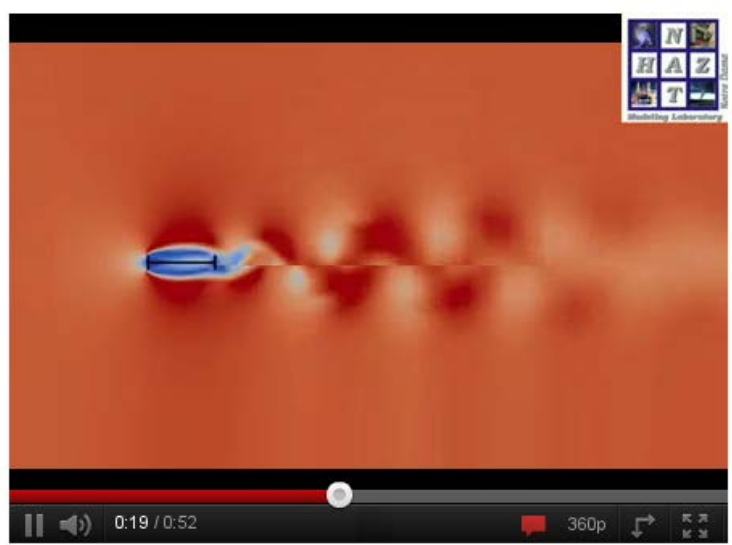

Fig. 10. Movie Tutorial For OpenFOAM Simulation

Such expert-citizens have unique goals and expectations that are different from average citizens, such as amateurs and hobbyists in traditional citizen science projects. To leverage the expertise of skilled citizens, we need to develop new principles and guidelines to achieve successful designs. These new guidelines may be different from the strategies of fulfilling tasks that require less experience.

Inspired by previous research on leveraging "citizen expert groups" to achieve common social scientific goals [14] [7, we identify the following 3 challenges that are unique to expert citizen engineering projects.

1. Task Complexity. In expert citizen projects, tasks usually demand high human intelligence and skill level. For example, experts can be solicited to provide insightful, informative and/or authoritative judgments.

2. Recruitment Difficulty. Due to the complexity inherent in tasks, available human resources are limited and membership eligibility is rather selective compared to traditional crowdsourcing tasks.

3. Resource Requirement. Complicated tasks may require sophisticated analysis tools and high performance computational resources [6]. For example, some analysis and design methods, such as nonlinear finite element analyses of complex structures, can easily over stress in-house computational capabilities of many firms and laboratories.

These challenges motivate us to investigate more effective engineering designs that can leverage expertise and experience afforded by highly skilled citizen engineers [28]. In this pilot project, highly skilled experts were invited to use a computational fluid dynamics (CFD) tool to analyze channel flow movements.

\subsection{OpenFOAM Package}

On the web platform, the primary simulation suite we provided is the OpenFOAM (Open Field Operation and Manipulation) Toolbox developed by OpenCFD Ltd 


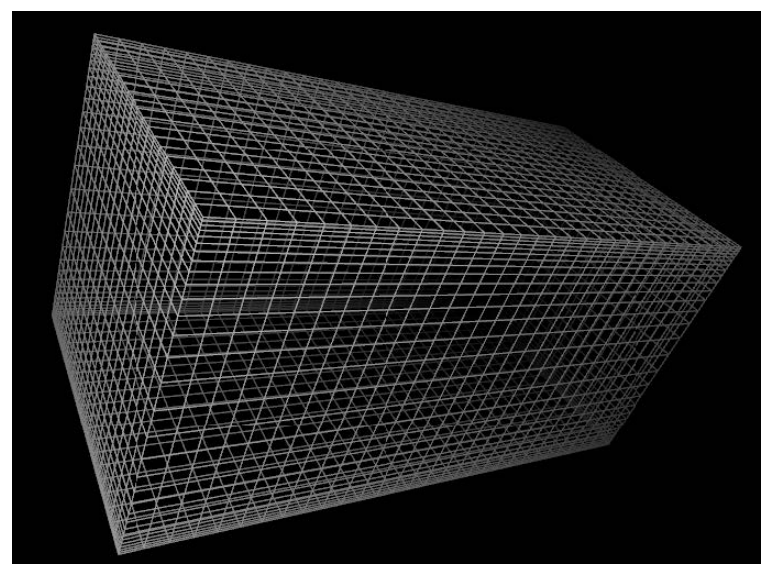

Fig. 11. Mesh Visualization of the Channel Flow. This screenshot is from one student's report.

4. The toolbox is an OSS package, licensed under the GNU General Public License (GPL). OpenFOAM's ability to simulate complex turbulence, and its openness to user customization and extension make itself one of most popular CFD simulation tools. It has been widely used by practitioners worldwide, and validated and verified intensively [4. Moreover, conveniently, OpenFOAM has an embedded meshing utility, which helps expert-citizens better present their results.

\subsection{Experiment Procedures}

The expert engineers in this pilot came from a graduate-level class - CE 80200 Wind Engineering - offered by the Department of Civil Engineering and Geological Sciences at Notre Dame. This senior graduate-level course covers primary design considerations under a variety of wind types. Topics include the analysis of structural response due to wind loading, modeling of wind-induced forces, and principles of resilient design under high wind loads. 9 graduate students and visiting scholars enrolled in the class; all had extensive formal training in civil engineering, equipped with knowledge of both civil engineering and OpenFOAM software.

\subsection{Workflow}

First, users were presented with a question set, in which questions were designed to test users' basic understanding of lecture materials. After the questions, users were taken to the main interface, Fig. 10, where they could receive the work assignment, review the previous documents, login to run the simulation platform and submit their results in the end. A sample mesh generation is shown in Fig. 11 


\subsection{Post-experiment Interview}

After the experiment was complete, we interviewed subjects who had first-hand experience about the platform. Most concerns were centered around the robustness of the simulation platform. When users were asked this question, "Please describe the difficulties you had using the simulation platform?", here are some representative responses:

- "The performance, error handling and reliability of the computing services could be improved."

- "Sometimes, I cannot proceed with my simulation because of the high traffic on the platform."

It is implied by users' concerns that when our web site has provided basic functionalities, expert citizens especially emphasize the reliability and stability of the system that can facilitate them to navigate through complicated tasks. In this regard, the retention of expert citizens to a large degree depends on the satisfaction of their high expectations on user experience. "Being usable and being likable are two different goals" 29. Easiness and smoothness plays a more primary role for professional users than it does for average users.

\section{Discussions and Future Work}

In this paper, we introduced 6 general categories of CE practices: Crowd Decisions, Crowd Submissions/Funding/Journalism, Crowd Wisdom, Crowd Byproduct, Micro Task, and Innovation Tournament. OSS development can be a form of crowd wisdom, where loosely connected online software engineers make large or small contributions over time, and the quality of the collective artifacts gradually get improved. Likewise, end users of OSS make contributions to the development through their feature requests, bug reports, and Q\&A on the project forums. We can apply the same approaches/principles proved successful in OSS development to other engineering domains. The 4 pilot projects presented in the previous sections attempt to generalize these rules beyond the OSS domain.

As always, more research problems emerged than were answered. For example, in Micro Task, what is the optimal number of users to work on the same task in order to secure a quality result? How should we rate and group online users based on their performance? In Crowd Wisdom, how can we efficiently divide the task into units and aggregate crowds' inputs later on, when the tasks are interdependent? How can we automate the verification and evaluation process? In Innovation Tournament, how can a competition be more appealing to those users with strong expertise and professional background? Higher monetary prize or stronger moral motivation in recognition? Appropriately answering these questions will help the future research and development of CE systems, more effectively leveraging the "wisdom of the crowd". 
Acknowledgment. The research presented in this paper was supported in part by an award from the National Science Foundation, under Grant No. CBET0941565. Any opinions, findings, and conclusions or recommendations expressed in this material are those of the author(s) and do not necessarily reflect the views of the National Science Foundation. The authors would also like to thank Zack Kertcher, Peter Sempolinski, Douglas Thain, Ellen Childs, Daniel Wei for valuable contributions.

\section{References}

1. American Society of Civil Engineers (ASCE). Report Card for America's Infrastructure (2009)

2. CENS, http://research.cens.ucla.edu/ (retrieved August 2011)

3. ImageCat, http://www.imagecatinc.com/ (retrieved August 2011)

4. OpenFOAM, http://www.openfoam.com/features/index.php (retrieved July 2011)

5. Global mobile cellular subscriptions, http://www.itu.int/ITU-D/ict/statistics/ (retrieved March 2012)

6. OSDCI, http://www.ne.edu/ opence/ (retrieved March 2012)

7. Ahlheim, M., Ekasingh, B., Frör, O., Kitchaincharoen, J., Neef, A., Sangkapitux, C., Sinphurmsukskul, N.: Using citizen expert groups in environmental valuation - Lessons from a CVM study in Northern Thailand. Technical Report 283/2007, Department of Economics, University of Hohenheim, Germany (2007)

8. Bennett, J., Lanning, S.: The Netflix prize. In: KDD Cup and Workshop in Conjunction with KDD (2007)

9. Bjelland, O.M., Chapman Wood, R.: An inside view of IBM's Innovation Jam. MIT Sloan Management Review 50(1), 32-40 (2008)

10. Brabham, D.C.: Crowdsourcing as a model for problem solving: An introduction and cases. Convergence 14(1), 75 (2008)

11. Burke, J., Estrin, D., Hansen, M., Praker, A., Ramanathan, N., Reddy, S., Srivastava, M.: Participatory sensing. In: ACM Sensys World Sensor Web Workshop, Boulder, CO, USA (October 2006)

12. Campbell, A.T., Eisenman, S.B., Lane, N.D., Miluzzo, E., Peterson, R.A., Lu, H., Zheng, X., Musolesi, M., Fodor, K., Ahn, G.-S.: The rise of people-centric sensing. IEEE Internet Computing 12, 12-21 (2008)

13. Doan, A., Ramakrishnan, R., Halevy, A.Y.: Crowdsourcing systems on the worldwide web. Communications of ACM 54(4) (April 2011)

14. Fischer, F.: Citizen participation and the democratization of policy expertise: From theoretical inquiry to practical cases. Policy Sciences 26, 165-187 (1993)

15. Goldman, J., Shilton, K., Burke, J., Estrin, D., Hansen, M., Ramanathan, N., Reddy, S., Samanta, V., Srivastava, M., West, R.: Participatory Sensing: A citizenpowered approach to illuminating the patterns that shape our world. Foresight \& Governance Project, White Paper (2009)

16. Goodchild, M.: Citizens as sensors: the world of volunteered geography. Geo Journal 69(4), 211-221 (2007)

17. Hedberg, H., Iivari, N.: Integrating HCI Specialists into Open Source Software Development Projects. In: Boldyreff, C., Crowston, K., Lundell, B., Wasserman, A.I. (eds.) OSS 2009. IFIP AICT, vol. 299, pp. 251-263. Springer, Heidelberg (2009) 
18. Kansal, A., Goraczko, M., Zhao, F.: Building a sensor network of mobile phones. In: Proceedings of the 6th International Conference on Information Processing in Sensor Networks, IPSN 2007, pp. 547-548. ACM, Cambridge (2007)

19. Kijewski-Correa, T., et al.: Open sourcing the design of civil infrastructure (OSDCI): A paradigm shift. In: Proceedings of Structures Congress, Las Vegas, NV, USA (April 2011)

20. Kittur, A., Chi, H., Suh, B.: Crowdsourcing user studies with mechanical turk. In: Proceedings of CHI 2008, pp. 453-456. ACM Press (2008)

21. Locke, E.A., Latham, G.P.: A theory of goal setting and task performance. PrenticeHall, Englewood Cliffs (1990)

22. Mavandadi, S., Dimitrov, S., Feng, S., Yu, F., Sikora, U., Yaglidere, O., Padmanabhan, S., Nielsen, K., Ozcan, A.: Distributed medical image analysis and diagnosis through crowd-sourced games: A malaria case study. PLoS ONE 7(5), e37245 (2012)

23. Mix, D., Kijewski-Correa, T., Taflanidis, A.A.: Assessment of residential housing in Leogane, Haiti after the January 2010 earthquake and identification of needs for rebuilding. In: Earthquake Spectra (October 2011)

24. Reddy, S., Mun, M., Burke, D.E.J., Hansen, M., Srivastava, M.: Using mobile phones to determine transportation modes. ACM Transactions on Sensor Networks 6(2) (February 2010)

25. Shirky, C.: Cognitive Surplus: Creativity and Generosity in a Connected Age. Allen Lane (2010)

26. Tang, J.C., Cebrian, M., Giacobe, N.A., Kim, H.-W., Kim, T., Wickert, D.B.: Reflecting on the DARPA Red Balloon Challenge. Communications of the ACM 54(4), 78-85 (2011)

27. Zhai, Z., Hachen, D., Kijewski-Correa, T., Shen, F., Madey, G.: Citizen engineering: Methods for "crowd sourcing" highly trustworthy results. In: Proceedings of the Forty-fifth Hawaii International Conference on System Science (HICSS-45), Maui, HI, USA, January 4-7 (2012)

28. Zhai, Z., Sempolinski, P., Thain, D., Madey, G.R., Wei, D., Kareem, A.: Expertcitizen engineering: "crowdsourcing" skilled citizens. In: DASC, pp. 879-886. IEEE (2011)

29. Zhang, P., von Dran, G.M.: Satisfiers and dissatisfiers: A two-factor model for website design and evaluation. Journal of the American Society for Information Science 51(14), 1253-1268 (2000) 